\title{
Single-Needle Lateral Sacroplasty Technique
}

\author{
(D).J. Nicholson, DC.A. Hilditch, (1)W. Brinjikji, DA.C.O. Tsang, and (D) R. Smith
}

\section{ABSTRACT}

SUMMARY: Sacral insufficiency fractures result in significant morbidity, and percutaneous sacroplasty has emerged as a promising technique for their treatment. We present a technical note regarding our method of treating these fractures using a "single-needle" lateral technique with a combination of conebeam CT and biplane fluoroscopy. We treated 10 patients, in whom the median Visual Analog Scale pain score decreased from 7.0 to $0(P<.001)$. We concluded that single-needle sacroplasty is feasible and safe using this technique.

$\mathbf{S}$ acral fractures can be secondary to osteoporosis (sacral insufficiency fractures) or malignancy, are relatively common, and can cause severe pain. This pain can be especially debilitating in elderly patients, in whom pain-related mobility impairment can have a detrimental effect on quality of life as well as predispose patients to a range of conditions associated with long-term immobility such as deep venous thrombosis and respiratory problems. Traditionally, these lesions are treated with bed rest, anesthesia, and physiotherapy. ${ }^{1}$ In the past decade, sacroplasty has emerged as an effective technique for the treatment of these painful lesions. ${ }^{2}$ This primarily stemmed from vertebral augmentation techniques, with the injection of polymethylmethacrylate cement. Since it was first described, sacroplasty has been shown to be effective in decreasing pain and improving quality of life. ${ }^{3}$

Even during the first description of the procedure, ${ }^{4}$ it was recognized that combining the benefits of CT and fluoroscopy would allow accurate needle tip positioning as well as real-time monitoring of cement injection. To this end, we use a biplane fluoroscopy machine equipped with conebeam CT capabilities (Allura Xper FD20/10; Philips Healthcare, Best, the Netherlands) for all of our sacroplasty procedures. In conjunction with needle-trajectoryplanning software (XperGuide; Philips Healthcare), this allows meticulous planning of the needle position and trajectory. Sacroplasty is commonly performed using multiple needles to cover

Received July 30, 2018; accepted after revision September 28.

From the Division of Neuroradiology (P.J.N., C.A.H., W.B., A.C.O.T., R.S.), Joint Department of Medical Imaging, Toronto Western Hospital, University of Toronto, Toronto, Ontario, Canada; and Division of Neurosurgery (A.C.O.T.), Department of Surgery, Queen Mary Hospital, University of Hong Kong, Hong Kong.

Please address correspondence to Patrick J. Nicholson, MD, Division of Neuroradiology, Joint Department of Medical Imaging, Toronto Western Hospital,

University of Toronto, 399 Bathurst St, Toronto, Ontario, M5T 2S8, Canada;

e-mail: paddynicholson@gmail.com

http://dx.doi.org/10.3174/ajnr.A5884 both the sacral ala and the central component of the bone. Each needle-placement site is associated with an individual risk of damage to nerves or vascular structures, along with a risk of infection and the inherent pain and soft-tissue swelling associated with each puncture. There is therefore an onus on the operator to deliver cement using as few delivery needles as possible. A percutaneous transiliac approach for stabilizing screw insertion has been used in orthopedic surgery. Thus, we began by using a long "single-needle" lateral technique, positioning the needle across the transverse axis of the sacrum (ie, from one sacral ala to the other). By beginning deposition of cement at this ala and then progressively withdrawing the needle and progressively injecting cement in continuity under real-time fluoroscopic guidance, the operator can successfully perform an excellent sacroplasty using a single needle, thereby avoiding the risk of complications outlined above. The transverse cement placement incorporates the support of the column of the central sacral body with the column of the ala.

\section{MATERIALS AND METHODS}

This study was performed with the approval of the research and ethics board of Toronto Western Hospital. A prospective data base was maintained, following all patients who underwent spinal and sacral augmentation procedures. From this, all patients who underwent sacroplasty using a single-needle technique were identified. All patients had undergone a preprocedural clinical assessment, followed by a clinic visit at 30 days following the procedure. Continuous variables were reported as mean (95\% confidence intervals), while Visual Analog Scale scores were reported as median (interquartile range). Pain Visual Analog Scale scores were also subjected to a paired $t$ test using GraphPad Prism software, Version 6.04 for Windows, (GraphPad Software, San Diego, California). 


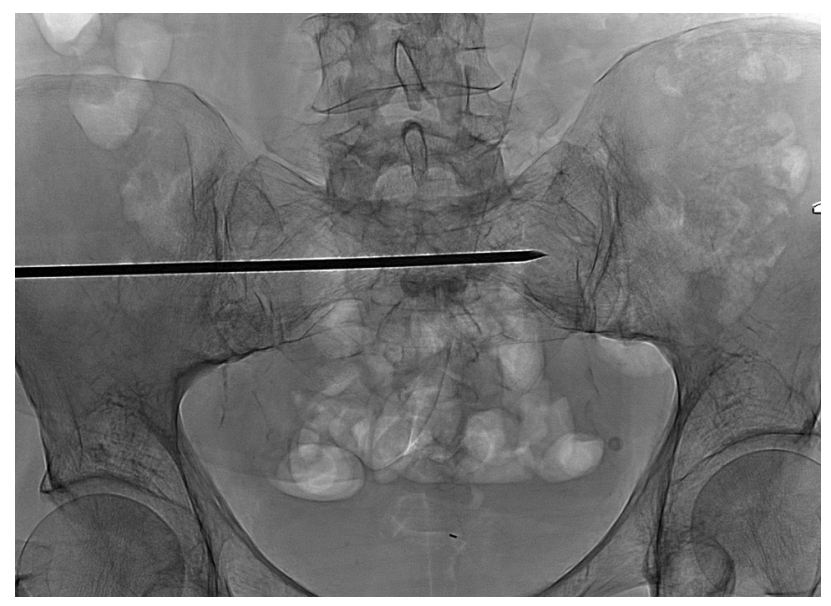

FIG 1. Posteroanterior fluoroscopic image showing the 11-ga needle inserted via the left sacral ala in a transiliac approach.

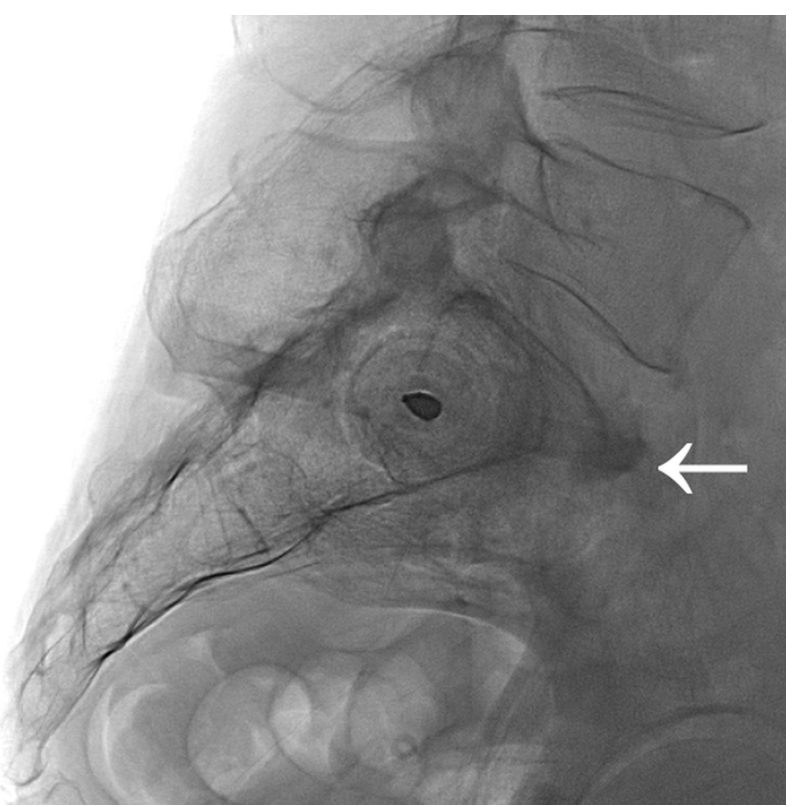

FIG 2. Lateral fluoroscopic image showing the same needle inserted into S1, positioned within the intramedullary cavity between the anterior and posterior cortices of the bone. Note an osteophyte anterior to the anterior margin of the sacrum (white arrow).

\section{Technique and Illustrative Case}

A 75-year-old woman had experienced painful sacral fractures (Fig 1). She had a history of multiple myeloma for which she had received radiation therapy to her spine and pelvis. Her mobility and quality of life were markedly impaired, to the extent that she required a walker and was using considerable amounts of opiate medication daily. She ranked her average daily pain as 7 on the Visual Analog Scale when assessed in the spinal clinic. The procedure was performed with the patient under conscious sedation provided by the anesthesiology service. The patient was positioned prone on the angiography table for the procedure, with straps used to minimize movement. Most important, a foam wedge was used to elevate the ipsilateral side of the patient (ie, the side of the entry point). The most common entry site is via the left sacral ala, which is the on the side of the operator when the patient is positioned prone. Conebeam CT was performed, and a needle

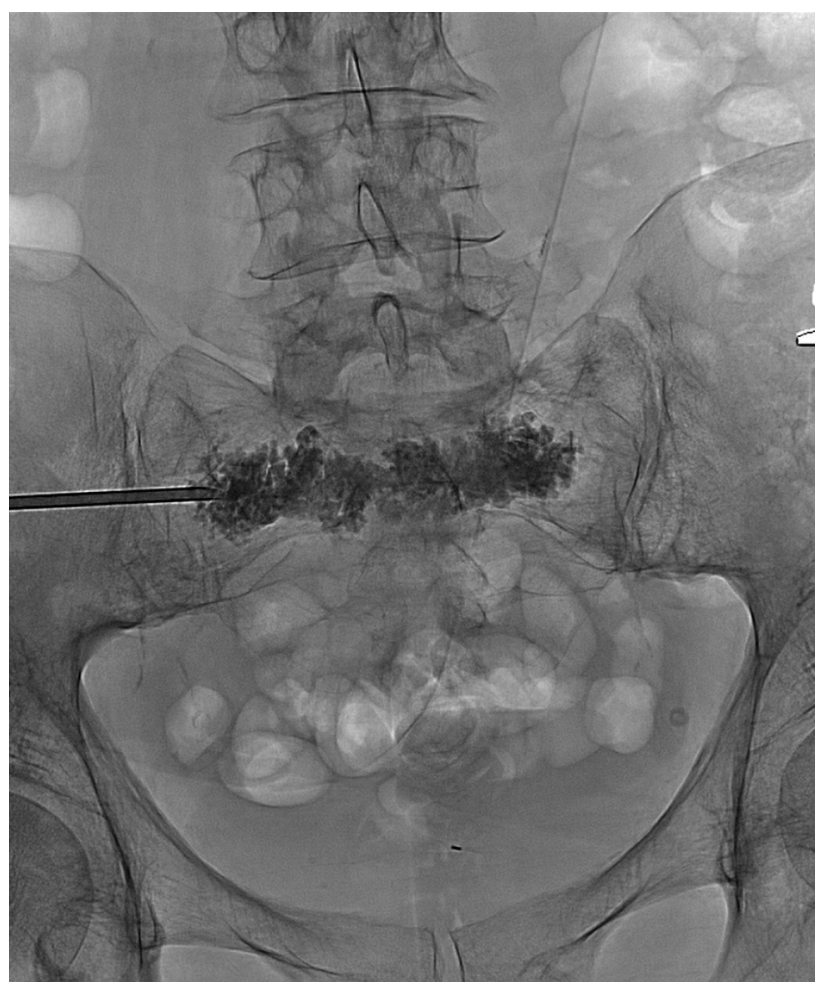

FIG 3. Posteroanterior fluoroscopic image showing the needle having been withdrawn in a transiliac fashion along its insertion path, injecting aliquots of cement as it is withdrawn.

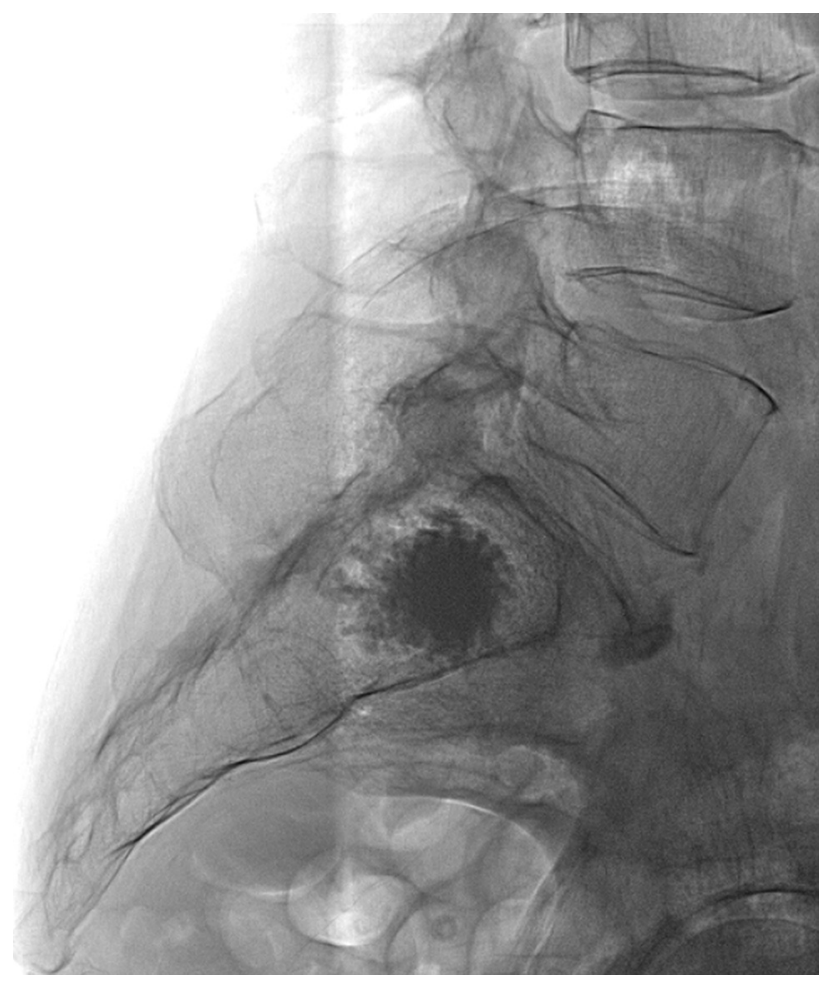

FIG 4. Lateral fluoroscopic image following needle withdrawal showing the polymethylmethacrylate cement confined to the sacral cortex.

trajectory was chosen on the planning software package (Fig 2) using XperGuide software. A $10-\mathrm{mL}$ mix of $1 \%$ lidocaine and $0.25 \%$ marcaine was injected in the subcutaneous tissues down to 


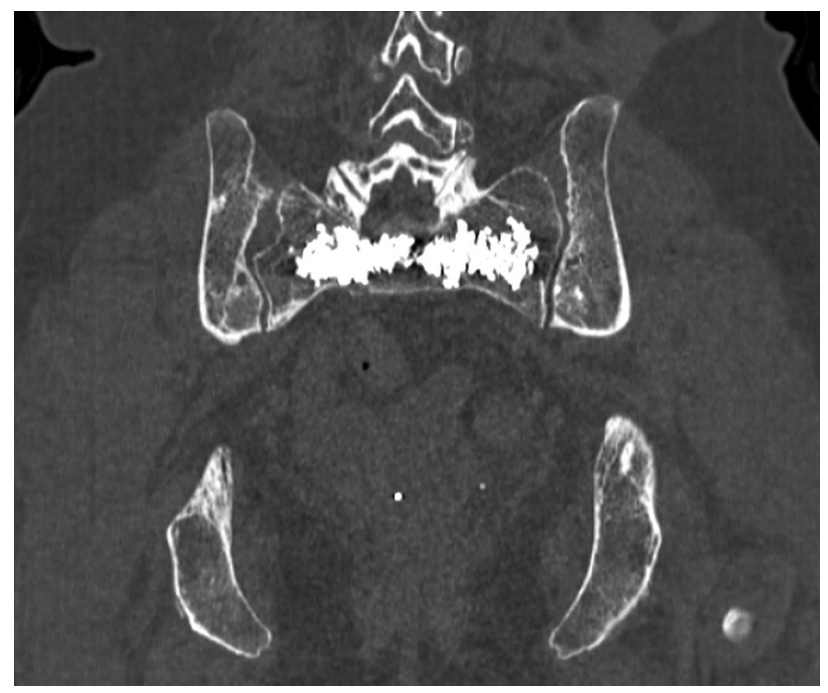

FIG 5. Axial reconstruction from a postoperative CT scan showing the cement well-positioned within the intramedullary cavity of the sacrum.

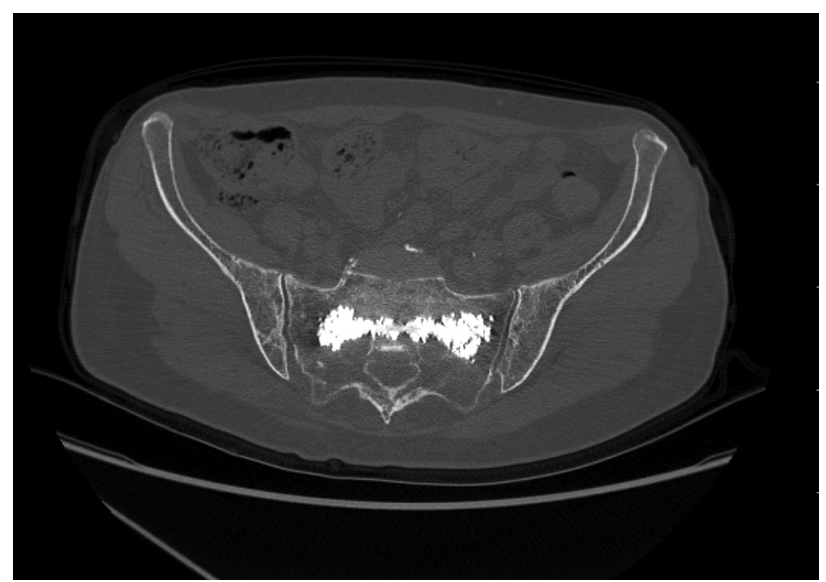

FIG 6. Coronal reconstruction from a postoperative CT scan showing the cement well-positioned within the intramedullary cavity of the sacrum.

the level of the periosteum. Following a skin incision, an 11-ga (15 $\mathrm{cm}$ ) diamond-tipped needle was used to perform the sacroplasty, while accessing the sacrum from the left side. The needle crossed the midline to the contralateral sacral ala (Figs 3 and 4 ). We injected $11 \mathrm{~mL}$ of cement using real-time biplane fluoroscopic guidance, injecting slowly in discrete aliquots of cement as the needle was withdrawn to the ipsilateral sacral ala via the midline sacral body. Continuous fluoroscopic monitoring for extrusion of cement outside the sacral bone was performed. The patient tolerated the procedure well and was ambulating again 1 hour after returning to the day surgery ward. At her 1-month follow-up visit, she reported that she was pain-free and no longer required opiates for anesthesia (Figs 5 and 6).

\section{RESULTS}

During a 1-year period, 10 patients underwent single-needle lateral sacroplasty using this technique. Their clinical details and outcome data are summarized in the Table. The median patient mean age was 67.5, IQR 12.75 (95\% CI, 57.8-77.2 years). Six of
Patient details and outcome data

\begin{tabular}{lcclcc}
\hline Sex & $\begin{array}{c}\text { Age } \\
\text { (yr) }\end{array}$ & $\begin{array}{c}\text { Cement } \\
\text { Volume } \\
\text { Injected } \\
\text { (mL) }\end{array}$ & $\begin{array}{c}\text { Primary } \\
\text { Disease }\end{array}$ & $\begin{array}{c}\text { Pain } \\
\text { Preprocedure } \\
\text { (VAS) }\end{array}$ & $\begin{array}{c}\text { Pain 1 } \\
\text { Month } \\
\text { Postprocedure } \\
\text { (VAS) }\end{array}$ \\
\hline Female & 36 & 14 & Breast carcinoma & 4 & 0 \\
Male & 59 & 11 & Myeloma & 7 & 2 \\
Female & 62 & 12 & $\begin{array}{c}\text { Non-small cell } \\
\text { lung carcinoma }\end{array}$ & 7 & 0 \\
Male & 66 & 25 & Myeloma & 5 & 0 \\
Male & 86 & 10 & Osteoporosis & 7 & 0 \\
Female & 69 & 10 & Osteoporosis & 10 & 6 \\
Female & 69 & 10 & Breast carcinoma & 8 & 4 \\
Female & 77 & 11 & Osteoporosis & 7 & 3 \\
Female & 75 & 11 & Myeloma & 7 & 0 \\
Male & 76 & 8 & Non-small cell & 8 & 0 \\
& \multicolumn{7}{c}{ lung carcinoma } & & \\
\hline
\end{tabular}

Note:-VAS indicates Visual Analog Scale.

the 10 patients were women. Fracture etiology was osteoporotic insufficiency in 3 patients (30\%), while the remainder had cancerrelated fractures (70\%). The median Visual Analog Scale score before the procedure was 7.0 (interquartile range, 7-7.5), while the median Visual Analog Scale score measured at 30-day follow-up was 0 (interquartile range, $0-2.75 ; P \leq 0.01$ ). One patient experienced severe right-leg pain in the $\mathrm{S} 1$ distribution the day after the procedure. This resolved following an epidural nerve root injection of steroid and local anesthesia, and she remained pain-free on follow-up.

\section{DISCUSSION}

Sacroplasty is emerging as a valuable tool for the treatment of sacral fractures, both insufficiency fractures and those related to cancer. ${ }^{5}$ While the technique shows great promise, there are many inherent challenges to overcome. These include the relatively complex anatomy of the sacral bone, as well as the need for accurate needle placement to avoid damage to either the surrounding pelvic organs or the neurovascular structures that course through the sacrum. Conventional radiographic views are therefore insufficient due to the relatively high associated rates of needle malposition. ${ }^{6}$ Angiography suites equipped with conebeam CT have allowed acquisition of volumetric CT images on the angiography table, thereby combining the advantages of traditional CT imaging guidance with those of real-time biplane fluoroscopy. These allow real-time high-resolution image reconstruction in any plane. When used with needle-tracking/planning software, this acquisition allows precise trajectory planning and real-time needle and cement-position monitoring. This biplane equipment or planning software or both are not strictly necessary; indeed, many groups perform this procedure using, for example, CT flouroscopy. However, we think that if it is available with your equipment, the use of biplane \pm planning software does offer benefit and it's use should be at least considered.

The classification of Denis et $\mathrm{al}^{7}$ of sacral insufficiency fractures organizes these fractures according to direction, location, and level. Most of these fractures occur in the craniocaudal direction, so the use of a lateral- or transverse-axis approach allows cement deposition across the fracture line, buttressing the fracture on either side.

The type of fracture and underlying pathology also guide our approach. A single-needle approach is possible in some but not all solid tumors; occasionally, a multiple-needle approach may be 


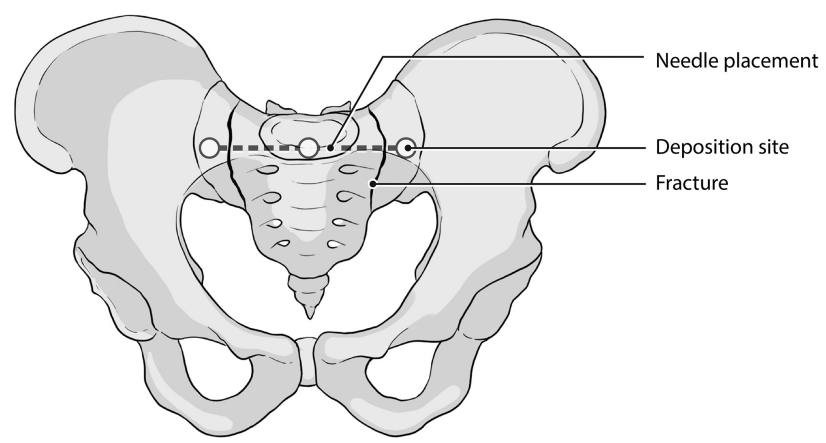

FIG 7. Illustration of the correct needle trajectory showing the needle path and method of cement deposition in the anteroposterior plane.

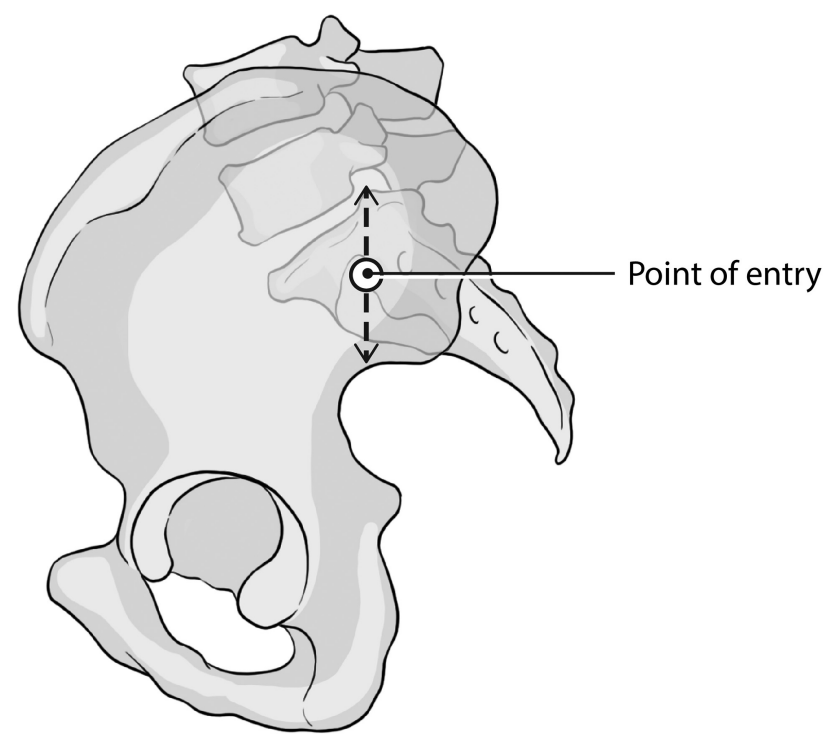

FIG 8. Illustration of the correct needle trajectory showing the needle path and method of cement deposition in the lateral plane.

necessary to target multiple solid lesions. A single-needle approach is more often feasible in patients with primary osteoporosis, secondary osteoporosis (eg, secondary to systemic treatment or pelvic irradiation), or diffuse disease (eg, multiple myeloma). We therefore carefully review the preoperative imaging and underling etiology and tailor our treatment approach accordingly.

The curved structure of the sacral bone means that traditional anteroposterior and lateral radiographic views are often insufficient to provide safe working angles for needle placement. ${ }^{8}$ Percutaneous screws have been used for fixation for posterior sacral fractures, ${ }^{9}$ and the technique has shown promise. Lateral sacroplasty emulates this approach, taking advantage of the lateral view to minimize needle and cement penetration through the anterior cortex of the bone (Figs 7 and 8). This transverse-axis approach usually means using 2 separate needles for both sacral alas, however. Our approach allows good cement filling of the intramedullary space of the sacrum using a single skin incision and bone puncture and, therefore, comes with inherently less risk of hematoma and infection. There is also less pain locally for the patient following the procedure.

\section{CONCLUSIONS}

Sacroplasty is emerging as a useful tool for the treatment of sacral fractures, either due to osteoporosis or cancer. We have presented our single-needle technique, which uses the advantages of ontable conebeam CT combined with real-time biplane fluoroscopic guidance. This allows a complete sacroplasty using only 1 needle, and in our series, it was associated with significant reduction in pain scores as measured by the Visual Analog Scale.

\section{REFERENCES}

1. Bydon M, Fredrickson M, De la Garza-Ramos R, et al. Sacral fractures. Neurosurg Focus 2014;37:E12 CrossRef

2. Pereira LP, Clarençon F, Cormier E, et al. Safety and effectiveness of percutaneous sacroplasty: a single-centre experience in 58 consecutive patients with tumours or osteoporotic insufficient fractures treated under fluoroscopic guidance. Eur Radiol 2013;23:2764-72 CrossRef Medline

3. Siggens K, Sabou S, Pasku D, et al. Systematic review of sacroplasty for metastases to the sacrum. Spine J 2016;16:S89

4. Pommersheim W, Huang-Hellinger F, Baker M, et al. Sacroplasty: a treatment for sacral insufficiency fractures. AJNR Am J Neuroradiol 2003;24:1003-07 Medline

5. Hirsch, JA, Barr JD, Zoarski GH. Sacroplasty: beyond the beginning. J Neurointerv Surg 2013;5:395 CrossRef Medline

6. Routt ML Jr, Simonian PT, Agnew SG, et al. Radiographic recognition of the sacral alar slope for optimal placement of iliosacral screws: a cadaveric and clinical study. J Orthop Trauma 1996;10:171-77 CrossRef Medline

7. Denis F, Davis S, Comfort T. Sacral fractures: an important problem-retrospective analysis of $\mathbf{2 3 6}$ cases. Clin Orthop Relat Res 1988; 227:67-81 Medline

8. Xu R, Ebraheim NA, Robke J, et al. Radiologic evaluation of iliosacral screw placement. Spine (Phila Pa 1976) 1996;21:582-88 CrossRef Medline

9. Suzuki T, Hak DJ, Ziran BH, et al. Outcome and complications of posterior transiliac plating for vertically unstable sacral fractures. Injury 2009;40:405-09 CrossRef Medline 